\title{
Neutrino Pair Cerenkov Radiation for Tachyonic Neutrinos
}

\author{
Ulrich D. Jentschura ${ }^{1,2}$ and István Nándori ${ }^{2}$ \\ ${ }^{1}$ Department of Physics, Missouri University of Science and Technology, Rolla, MO 65409, USA \\ ${ }^{2}$ MTA-DE Particle Physics Research Group, P.O. Box 51, Debrecen 4001, Hungary \\ Correspondence should be addressed to Ulrich D. Jentschura; ulj@mst.edu
}

Received 25 May 2017; Accepted 17 October 2017; Published 12 November 2017

Academic Editor: Ming Liu

Copyright (C) 2017 Ulrich D. Jentschura and István Nándori. This is an open access article distributed under the Creative Commons Attribution License, which permits unrestricted use, distribution, and reproduction in any medium, provided the original work is properly cited. The publication of this article was funded by SCOAP ${ }^{3}$.

\begin{abstract}
The emission of a charged light lepton pair by a superluminal neutrino has been identified as a major factor in the energy loss of highly energetic neutrinos. The observation of PeV neutrinos by IceCube implies their stability against lepton pair Cerenkov radiation. Under the assumption of a Lorentz-violating dispersion relation for highly energetic superluminal neutrinos, one may thus constrain the Lorentz-violating parameters. A kinematically different situation arises when one assumes a Lorentz-covariant, space-like dispersion relation for hypothetical tachyonic neutrinos, as an alternative to Lorentz-violating theories. We here discuss a hitherto neglected decay process, where a highly energetic tachyonic neutrino may emit other (space-like, tachyonic) neutrino pairs. We find that the space-like dispersion relation implies the absence of a $q^{2}$ threshold for the production of a tachyonic neutrinoantineutrino pair, thus leading to the dominant additional energy loss mechanism for an oncoming tachyonic neutrino in the medium-energy domain. Surprisingly, the small absolute values of the decay rate and energy loss rate in the tachyonic model imply that these models, in contrast to the Lorentz-violating theories, are not pressured by the cosmic PeV neutrinos registered by the IceCube collaboration.
\end{abstract}

\section{Introduction}

After early attempts at the construction of tachyonic neutrino theories [1-7], progress in the theoretical development was hindered by difficulties in the construction of a viable field theory involving tachyons (a particularly interesting argument was presented in [8]). Despite the difficulties, work on tachyonic theories has continued up to this day, for both classical theories and spin-zero and spin-1/2 quantum theories [9-12]. A very interesting hypothesis was brought forward by Chodos et al. [13], who developed a tachyonic neutrino model based on the so-called tachyonic Dirac equation. They recognized that a simple modification of the mass term in the Dirac equation, according to the replacement $m \rightarrow \gamma^{5} m$, induces a dispersion relation of the form $E=\sqrt{\vec{p}^{2}-m^{2}}$ (with the "tachyonic" sign in front of the mass term), while preserving the spin- $1 / 2$ character of the equation. Recently, it has been recognized [14] that the modified Dirac Hamiltonian corresponding to the tachyonic solutions has a property known as pseudo-Hermiticity, which has been recognized as a viable generalization of the concept of Hermiticity, for quantum mechanical systems [15-25]. Furthermore, the bispinor solutions of the tachyonic equation have been determined [26], and they have been shown to fulfill sum rules which enter the calculation of the timeordered product of tachyonic field operators. The tachyonic pseudo-Hermitian quantum dynamics of wave packets composed of the bispinor solutions has been discussed in [14]. A surprising feature of the tachyonic Dirac equation is the natural appearance of the fifth current in the equation. In particular, the appearance of $\gamma^{5}$ elevates the helicity basis to the most natural ansatz for the solution of the equation and induces parity-breaking in a natural way. States with the "wrong helicity" are eliminated from the theory by a GuptaBleuler type condition [26].

Just to fix ideas, we should point out here that the tachyonic neutrino differs from other faster-than-light neutrino models in that the dispersion relation is Lorentz-covariant. Explicit breaking of the Lorentz symmetry may induce fasterthan-light dynamics for neutrino wave packets, with a timelike four-vector product $p^{\mu} p_{\mu}>0$ (see [27, 28]). An example is the Lorentz-breaking dispersion relation $E=|\vec{p}| v$ with 
$v>1$ (units with $\hbar=c=\epsilon_{0}$ are used throughout this paper). This dispersion relation follows [29, 30] from a Lorentzviolating "metric" $\widetilde{g}_{\mu \nu}=\operatorname{diag}(1,-v,-v,-v)$. A quite illuminating analysis of the model dependence of the calculation [29], with reference to conceivably different forms of the interaction Lagrangian, is given in [30]. By contrast, the tachyonic theory implies a space-like four-vector product $p^{\mu} p_{\mu}=-m^{2}<0$, thus leaving Lorentz symmetry intact and enabling the construction of bispinor solutions in the helicity basis [26].

Despite some "seductive" observations regarding the tachyonic neutrino model (most of all, pseudo-Hermiticity and natural emergence of the helicity eigenstates, as well as the suppression of states with the "wrong" helicity), any alternative neutrino model must also pass various other tests concerning the stability of highly energetic neutrinos against the emission of particle-antiparticle pairs. The IceCube collaboration has registered "big bird," an $E_{v}=(2.004$ $\pm 0.236) \mathrm{PeV}$ highly energetic neutrino $[31,32]$. If neutrinos in this energy range are stable against lepton pair Cerenkov radiation, then this sets rather strict bounds on the values of the Lorentz-violating parameters $[33,34]$. In a recent paper, lepton pair Cerenkov radiation has been analyzed as an energy loss mechanism for high-energy tachyonic neutrinos [35]. The kinematics in this case implies that the oncoming, decaying neutrino decays into a tachyonic state of lower energy, emitting an electron-positron pair (see Figure 1(a)). For the creation of an electron-positron pair, the threshold momentum for the virtual $Z^{0}$ boson is $q^{2}=4 m_{e}^{2}$, where $m_{e}$ is the electron mass.

For both the Lorentz-violating and the tachyonic neutrino models, one has not yet considered the additional decay and energy loss channel which proceeds via a virtual $Z^{0}$ boson and has a neutrino-antineutrino pair (as opposed to an electron-positron pair) in the exit channel (see Figure 1(b)). This process is not parametrically suppressed in comparison to the one with electrically charged particles in the exit channel, because of the weakly rather than electromagnetically interacting virtual particle $\left(Z^{0}\right.$ boson) in the middle. For the Lorentz-violating theories, the kinematics in this case becomes involved because one has to implement Lorentzviolating parameters for all four particle in the process: (i) the oncoming and exiting neutrino and (ii) the created neutrino-antineutrino pair. Previous studies [29, 30] have rather concentrated on the lepton pair Cerenkov radiation process as the dominant energy loss mechanism than the neutrino pair Cerenkov radiation; the kinematics in this case appears to be a lot easier to analyze than for neutrino pair Cerenkov radiation.

For the tachyonic case, one needs to calculate the process of neutrino-antineutrino pair Cerenkov radiation in full tachyonic kinematics, for both the in and out states. In particular, it is necessary to generalize the pair production threshold to the creation of a tachyonic neutrinoantineutrino pair. We organize this paper as follows. In Section 2, we derive the kinematic conditions for tachyonantitachyon pair production. The calculation of the threshold conditions and the energy loss mechanism for neutrino pair
Cerenkov radiation proceeds in Section 3. Consequences for tachyonic neutrino theories are summarized in Section 4.

\section{Pair Production Threshold}

For two tardyonic ("normal") particles of mass $m_{e}$, pair production threshold is reached when the pair is emitted collinearly, with two four-vectors $p^{\mu}=(E, \vec{k})=\left(E_{1}, \vec{k}_{1}\right)=$ $\left(E_{2}, \vec{k}_{2}\right)$ that fulfill

$$
\begin{aligned}
E & =\sqrt{\vec{k}^{2}+m_{e}^{2}} \\
q^{2} & =4 p^{\mu} p_{\mu}=4\left(\vec{k}^{2}+m_{e}^{2}\right)-4 \vec{k}^{2}=4 m_{e}^{2}
\end{aligned}
$$

The situation is completely different for the production of a tachyonic pair. Here, a well-defined lower threshold for $q^{2}$ is missing. For example, we have for the collinear pair with tachyonic mass parameter $E^{2}-\vec{k}^{2}=-m_{v}^{2}$, and $p^{\mu}=(E, \vec{k})=$ $\left(E_{1}, \vec{k}_{1}\right)=\left(E_{2}, \vec{k}_{2}\right)$,

$$
\begin{aligned}
E & =\sqrt{\vec{k}^{2}-m_{\mu}^{2}}, \\
q^{2} & =4 p^{\mu} p_{\mu}=4\left(\vec{k}^{2}-m_{\mu}^{2}\right)-4 \vec{k}^{2}=-4 m_{\mu}^{2},
\end{aligned}
$$

which is negative. For two neutrinos of different energy, emitted collinearly $\left(\vec{k}_{1}=k_{1} \widehat{\mathrm{e}}_{z}\right.$ and $\left.\vec{k}_{2}=k_{2} \widehat{\mathrm{e}}_{z}\right)$, one has

$$
\begin{aligned}
& E_{1}=\sqrt{k_{1}^{2}-m_{\mu}^{2}}, \\
& E_{2}=\sqrt{k_{2}^{2}-m_{\mu}^{2}}, \\
& q^{2}=\left(\sqrt{k_{1}^{2}-m_{\mu}^{2}}+\sqrt{k_{2}^{2}-m_{\mu}^{2}}\right)^{2}-\left(k_{1}+k_{2}\right)^{2} .
\end{aligned}
$$

In the limit of a small tachyonic mass parameter $m_{v}$, a Taylor expansion of the latter term leads to the expression

$$
q^{2}=-\left(2+\frac{k_{1}}{k_{2}}+\frac{k_{2}}{k_{1}}\right) m_{v}^{2}+\mathcal{O}\left(m_{v}^{4}\right) .
$$

In the limits $k_{1} \rightarrow 0, k_{2} \rightarrow \infty$ or alternatively $k_{1} \rightarrow \infty$, $k_{2} \rightarrow 0$, the latter expression may assume very large negative numerical values (see also Figure 2). There is thus no lower threshold for tachyonic pair production, expressed in $q^{2}$.

One might ask if arbitrarily large $q^{2}$ are compatible with the relativistic tachyonic pair production kinematics. In order to answer this question, we consider the production of an anti-collinear pair,

$$
\begin{aligned}
& \vec{k}_{1}=k_{1} \widehat{\mathrm{e}}_{z}, \\
& \vec{k}_{2}=-k_{2} \widehat{\mathrm{e}}_{z},
\end{aligned}
$$




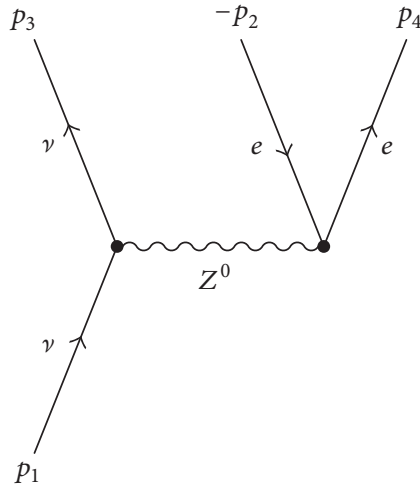

(a)

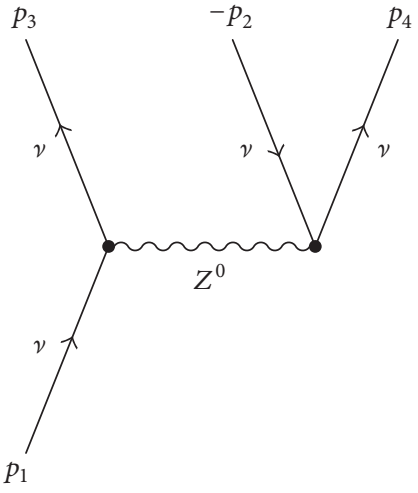

(b)

FIGURE 1: The decay process in (a) involves a faster-than-light neutrino decaying into an electron-positron pair, with a threshold $q^{2}=4 m_{e}^{2}$ for the virtual $Z^{0}$ boson. In (b), a neutrino-antineutrino pair is emitted from an oncoming energetic neutrino. Both processes are kinematically allowed if one assumes that neutrinos are tachyons described by a dispersion relation of the form $p^{\mu} p_{\mu}=E^{2}-\vec{k}^{2}=-m_{v}^{2}$.

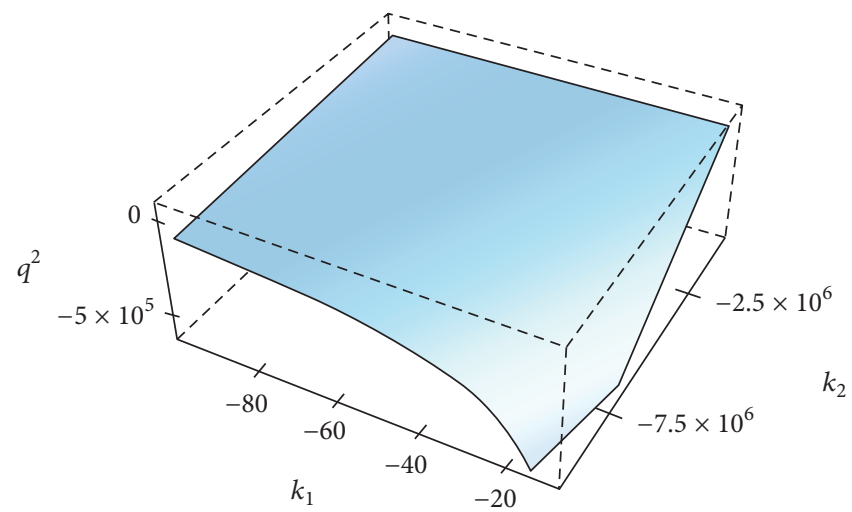

FIGURE 2: Plot of $q^{2}$ given in (3b) for tachyonic pair production $\left(m_{v}=1\right)$ in the region $-100<k_{1}<0$ and $-10^{7}<k_{2}<0$, demonstrating that $q^{2}$ may become large and negative even for small tachyonic mass parameters, when one of the momenta is significantly larger than the other (see (4)).

$$
\begin{aligned}
E_{1} & =\sqrt{k_{1}^{2}-m_{\mu}^{2}}, \\
E_{2} & =\sqrt{k_{2}^{2}-m_{\mu}^{2}} \\
q^{2} & =\left(\sqrt{k_{1}^{2}-m_{\mu}^{2}}+\sqrt{k_{2}^{2}-m_{\mu}^{2}}\right)^{2}-\left(k_{1}-k_{2}\right)^{2} \\
& =4 k_{1} k_{2}+\mathcal{O}\left(m_{\nu}^{2}\right) .
\end{aligned}
$$

For large $k_{1}$ and $k_{2}$, this expression assumes arbitrarily large positive numerical values.

The conclusion is that the tachyonic kinematics do not exclude any range of $q^{2}$ from the kinematically allowed range of permissible momentum transfers, and neutrino pair Cerenkov radiation (or, more generally, tachyon-antitachyon pair production) is allowed in the entire range

$$
\begin{gathered}
-\infty<q^{2}<\infty, \\
q^{0}>0 .
\end{gathered}
$$

The latter condition only ensures that the energy emitted into the pair is positive. For a decaying tachyonic neutrino, condition (6) implies that there is no lower energy threshold for the production of a tachyon-antitachyon pair from an oncoming neutrino, within the process depicted in Figure 1(b).

\section{Calculation of the Pair Production}

We calculate the decay width of the incoming tachyonic neutrino, in the lab frame, employing a relativistically covariant (tachyonic) dispersion relation, with both incoming and outgoing neutrinos on the tachyonic mass shell $\left(E_{i}=\right.$ $\sqrt{\vec{k}_{i}^{2}-m_{v}^{2}}$ for $\left.i=1,2,3,4\right)$, in the conventions of Figure 1 . In the lab frame, the decay rate is

$$
\begin{aligned}
\Gamma= & \frac{1}{2 E_{1}} \int \frac{\mathrm{d}^{3} p_{3}}{(2 \pi)^{3} 2 E_{3}}\left(\int \frac{\mathrm{d}^{3} p_{2}}{(2 \pi)^{3} 2 E_{2}} \int \frac{\mathrm{d}^{3} p_{4}}{(2 \pi)^{3} 2 E_{4}}\right. \\
& \times(2 \pi)^{4} \delta^{(4)}\left(p_{1}-p_{3}-p_{2}-p_{4}\right)\left[\widetilde{\left.\left.\sum_{\text {spins }}|M|^{2}\right]\right) .}\right.
\end{aligned}
$$


Here, $\widetilde{\Sigma}_{\text {spins }}$ refers to the specific way in which the average over the oncoming helicity states, and the outgoing helicities, needs to be carried out for tachyons [35].

We use the Lagrangian

$$
\mathscr{L}=-\frac{g_{w}}{4 \cos \theta_{W}}\left[\bar{\nu} \gamma^{\mu}\left(1-\gamma^{5}\right) \nu\right] Z_{\mu},
$$

where $\theta_{W}$ is the Weinberg angle, $Z_{\mu}$ is the $Z^{0}$ boson field, and $v$ is the neutrino field. The effective four-fermion interaction is

$$
\mathscr{L}=\frac{G_{F}}{2 \sqrt{2}}\left[\bar{\nu} \gamma^{\mu}\left(1-\gamma^{5}\right) \nu\right]\left[\bar{\nu} \gamma^{\mu}\left(1-\gamma^{5}\right) \nu\right],
$$

where $G_{F}$ is the Fermi coupling constant. The matrix element $\mathscr{M}$ is

$$
\begin{aligned}
\mathscr{M}= & \frac{G_{F}}{2 \sqrt{2}}\left[\bar{u}^{\mathscr{T}}\left(p_{3}\right) \gamma_{\lambda}\left(1-\gamma^{5}\right) u^{\mathscr{T}}\left(p_{1}\right)\right] \\
& \times\left[\bar{u}^{\mathscr{T}}\left(p_{4}\right) \gamma^{\lambda}\left(1-\gamma^{5}\right) v^{\mathscr{T}}\left(p_{2}\right)\right],
\end{aligned}
$$

where $u^{\mathscr{T}}(p)$ is a tachyonic positive-energy bispinor (particle) solution, while $v^{\mathscr{T}}(p)$ is a tachyonic negative-energy (antiparticle) solution. The positive-energy solutions read as follows [26]:

$$
\begin{aligned}
& u_{+}^{\mathscr{T}}(\vec{k})=\left(\begin{array}{c}
\sqrt{|\vec{k}|+m a_{+}}(\vec{k}) \\
\sqrt{|\vec{k}|-m a_{+}}(\vec{k})
\end{array}\right), \\
& u_{-}^{\mathscr{T}}(\vec{k})=\left(\begin{array}{c}
\sqrt{|\vec{k}|-m a_{-}}(\vec{k}) \\
-\sqrt{|\vec{k}|+m a_{-}}(\vec{k})
\end{array}\right),
\end{aligned}
$$

while the negative-energy solutions are given by

$$
\begin{aligned}
& v_{+}^{\mathscr{T}}(\vec{k})=\left(\begin{array}{c}
-\sqrt{|\vec{k}|-m a_{+}}(\vec{k}) \\
-\sqrt{|\vec{k}|+m a_{+}}(\vec{k})
\end{array}\right), \\
& v_{-}^{\mathscr{T}}(\vec{k})=\left(\begin{array}{c}
-\sqrt{|\vec{k}|+m a_{-}}(\vec{k}) \\
\sqrt{|\vec{k}|-m a_{-}}(\vec{k})
\end{array}\right),
\end{aligned}
$$

where we identify the on-shell spinors $u^{\mathscr{T}}(p)$ with the $u^{\mathscr{T}}(\vec{k})$, where $p^{\mu}=(E, \vec{k})$ and $E=\sqrt{\vec{k}^{2}-m_{v}^{2}}$. The symbols $a_{ \pm}(\vec{k})$ denote the fundamental helicity spinors (see p. 87 of [36]). We note that the helicity of the antineutrino solution $v_{-}^{\mathscr{T}}(\vec{k})$ is positive, while in the massless limit, it has negative chirality.

For the tachyonic spin sums, one has the following sum rule for the positive-energy spinors [37],

$$
\begin{aligned}
\sum_{\sigma} & (-\sigma) u_{\sigma}^{\mathscr{T}}(\vec{k}) \otimes \bar{u}_{\sigma}^{\mathscr{T}}(\widehat{k}) \gamma^{5} \\
\quad & =\sum_{\sigma}(-\vec{\Sigma} \cdot \widehat{k}) u_{\sigma}^{\mathscr{T}}(\vec{k}) \otimes \bar{u}^{\mathscr{T}}(\vec{k}) \gamma^{5}=\not p-\gamma^{5} m,
\end{aligned}
$$

where $\widehat{k}=\vec{k} /|\vec{k}|$ is the unit vector in the $\vec{k}$ direction. Upon promotion to a four-vector, one has $\widehat{k}^{\mu}=(0, \widehat{k})$. The sum rule can thus be reformulated as

$$
\begin{aligned}
\sum_{\sigma} u_{\sigma}^{\mathscr{T}}(p) \otimes \bar{u}_{\sigma}^{\mathscr{T}}(p) & =(-\vec{\Sigma} \cdot \widehat{k})\left(\not p-\gamma^{5} m_{\nu}\right) \gamma^{5} \\
& =-\gamma^{5} \gamma^{0} \gamma^{i} \widehat{k}^{i}\left(\not p-\gamma^{5} m_{\nu}\right) \gamma^{5} \\
& =-\not \gamma^{5} \hat{k}\left(\not p-\gamma^{5} m_{\nu}\right) \gamma^{5}
\end{aligned}
$$

where $\tau=(1,0,0,0)$ is a time-like unit vector.

In $[26,37]$, it has been argued that a consistent formulation of the tachyonic propagator is achieved when we postulate that the right-handed neutrino states and the left-handed antineutrino states acquire a negative Fock-space norm after quantization of the tachyonic spin-1/2 field. Hence, in order to calculate the decay process of an oncoming, left-handed, positive-energy neutrino, we must first project onto negativehelicity states, according to [35],

$$
\begin{aligned}
& \frac{1}{2}(1-\vec{\Sigma} \cdot \hat{k}) \sum_{\sigma} u_{\sigma}^{\mathscr{T}}(\vec{k}) \otimes \bar{u}_{\sigma}^{\mathscr{T}}(\vec{k}) \\
& \quad=u_{\sigma=-1}(p) \otimes \bar{u}_{\sigma=-1}(p) \\
& \quad=\frac{1}{2}\left(1-\not \gamma^{5} \hat{k}\right)\left(\not p-\gamma^{5} m_{\nu}\right) \gamma^{5} .
\end{aligned}
$$

The squared and spin-summed matrix element for the tachyonic decay process thus is

$$
\widetilde{\sum_{\text {spins }}}|\mathscr{M}|^{2}=\frac{G_{F}^{2}}{8} T_{13} T_{24}=\frac{G_{F}^{2}}{8} \mathcal{S}\left(p_{1}, p_{2}, p_{3}, p_{4}\right)
$$

where the latter identity provides an implicit definition of the function $\mathcal{S}\left(p_{1}, p_{2}, p_{3}, p_{4}\right)$. The traces $T_{13}$ and $T_{24}$ are

$$
\begin{aligned}
T_{13} & =\operatorname{Tr}\left[\frac{1}{2}\left(1-\not t \gamma^{5} \vec{k}_{3}\right)\left(\not p_{3}-\gamma^{5} m_{\nu}\right) \gamma^{5} \gamma_{\lambda}\left(1-\gamma^{5}\right)\right. \\
\times & \left.\frac{1}{2}\left(1-\not \gamma^{5} \vec{k}_{1}\right)\left(\not p_{1}-\gamma^{5} m_{\nu}\right) \gamma^{5} \gamma_{\nu}\left(1-\gamma^{5}\right)\right], \\
T_{24} & =\operatorname{Tr}\left[\frac{1}{2}\left(1-\not \gamma^{5} \vec{k}_{4}\right)\left(\not p_{4}-\gamma^{5} m_{\nu}\right) \gamma^{5} \gamma^{\lambda}\left(1-\gamma^{5}\right)\right. \\
& \left.\times \frac{1}{2}\left(1-\not \gamma^{5} \vec{k}_{2}\right)\left(\not p_{2}+\gamma^{5} m_{\nu}\right) \gamma^{5} \gamma_{\lambda}\left(1-\gamma^{5}\right)\right] .
\end{aligned}
$$

We have chosen the convention to denote the momentum of the outgoing antiparticle by $p_{2}$.

For the outgoing pair, we use the fact that the helicity projector is approximately equal to the chirality projector in the high-energy limit, which simplifies the Dirac gamma trace somewhat. On the tachyonic mass shell, one has $p_{1}^{2}=$ $p_{2}^{2}=p_{3}^{2}=p_{4}^{2}=-m_{v}^{2}$. After the trace over the Dirac $\gamma$ matrices, some resultant scalar products vanish, for example, the scalar product of the time-like unit vector $\tau$ and the spacelike unit vector $(\tau \cdot \widehat{k}=0)$. 
The result of the Dirac $\gamma$ traces from (15) is inserted into (7), and $\mathrm{d}^{3} p_{2}$ and $\mathrm{d}^{3} p_{4}$ integrals are carried out using the following formulas:

$$
\begin{aligned}
& I(q)=\int \frac{\mathrm{d}^{3} p_{2}}{2 E_{2}} \int \frac{\mathrm{d}^{3} p_{4}}{2 E_{4}} \delta^{(4)}\left(q-p_{2}-p_{4}\right)=\frac{\pi}{2} \\
& \cdot \sqrt{1+\frac{4 m_{v}^{2}}{q^{2}}}, \\
& J_{\lambda \rho}(q)=\int \frac{\mathrm{d}^{3} p_{2}}{2 E_{2}} \int \frac{\mathrm{d}^{3} p_{4}}{2 E_{4}} \delta^{(4)}\left(q-p_{2}-p_{4}\right)\left(p_{2 \lambda} p_{4 \rho}\right) \\
& \quad=\sqrt{1+\frac{4 m_{v}^{2}}{q^{2}}}\left[g_{\lambda \rho} \frac{\pi}{24}\left(q^{2}+4 m_{v}^{2}\right)\right. \\
& \left.+q_{\lambda} q_{\rho} \frac{\pi}{12}\left(1-\frac{2 m_{v}^{2}}{q^{2}}\right)\right], \\
& K(q)=\int \frac{\mathrm{d}^{3} p_{2}}{2 E_{2}} \int \frac{\mathrm{d}^{3} p_{4}}{2 E_{4}} \delta^{(4)}\left(q-p_{2}-p_{4}\right)\left(p_{2} \cdot p_{4}\right) \\
& \quad=\frac{\pi}{4} \sqrt{1+\frac{4 m_{v}^{2}}{q^{2}}}\left(q^{2}+2 m_{v}^{2}\right) .
\end{aligned}
$$

After $d^{3} p_{2}$ and $d^{3} p_{4}$ integrations, we are left with an expression of the form

$$
\Gamma=\frac{G_{F}^{2}}{8} \frac{1}{(2 \pi)^{5}} \int_{q^{2}>4 m_{e}^{2}} \frac{\mathrm{d}^{3} p_{3}}{2 E_{3}} \mathscr{F}\left(p_{1}, p_{3}\right),
$$

where

$$
\begin{aligned}
\mathscr{F}\left(p_{1}, p_{3}\right)= & \int \frac{\mathrm{d}^{3} p_{2}}{2 E_{2}} \int \frac{\mathrm{d}^{3} p_{4}}{2 E_{4}} \delta^{(4)}\left(p_{1}-p_{2}-p_{3}-p_{4}\right) \\
& \times \mathcal{S}\left(p_{1}, p_{2}, p_{3}, p_{4}\right) .
\end{aligned}
$$

Both the expressions for $\mathcal{S}\left(p_{1}, p_{2}, p_{3}, p_{4}\right)$ and $\mathscr{F}\left(p_{1}, p_{3}\right)$ are too lengthy to be displayed in the context of the current paper.

For the kinematics, we assume that

$$
\begin{aligned}
p_{1}^{\mu} & =\left(E_{1}, 0,0, k_{1}\right), \\
p_{3}^{\mu} & =\left(E_{3}, k_{3} \sin \theta \cos \varphi, k_{3} \sin \theta \sin \varphi, k_{3} \cos \theta\right), \\
E_{3}^{2}-k_{3}^{2} & =-m_{v}^{2}, \quad k_{3}>m_{v} .
\end{aligned}
$$

The condition $k_{3}>m_{v}$ is naturally imposed for tachyonic kinematics. The squared four-momentum transfer then reads as

$$
\begin{aligned}
q^{2} & =2\left(\sqrt{E_{1}^{2}+m_{\nu}^{2}} \sqrt{E_{3}^{2}+m_{\nu}^{2}} \cos \theta-E_{1} E_{3}-m_{\nu}^{2}\right) \\
& =2\left(k_{1} k_{3} u-\sqrt{k_{1}^{2}-m_{\nu}^{2}} \sqrt{k_{3}^{2}-m_{\nu}^{2}}-m_{\nu}^{2}\right),
\end{aligned}
$$

where it is convenient to define $u=\cos \theta$.
The integrations are done with the kinematic conditions that all $0<E_{3}<E_{1}$ and all $q^{2}=\left(p_{2}+p_{4}\right)^{2}$ for the pair are allowed (see Section 2), leading to

$$
\begin{aligned}
\Gamma & =\frac{G_{F}^{2}}{8} \frac{1}{(2 \pi)^{5}} \int_{0}^{2 \pi} \mathrm{d} \varphi \int_{k_{3}=m_{v}}^{k_{\max }} \frac{\mathrm{d} k_{3} k_{3}^{2}}{2 E_{3}} \\
& \cdot \int_{-1}^{1} \mathrm{~d} u \mathscr{F}\left(E_{1}, E_{3}, u\right)=\frac{G_{F}^{2}}{16} \frac{1}{(2 \pi)^{4}} \int_{0}^{E_{1}} \mathrm{~d} E_{3} \\
& \cdot \sqrt{E_{3}^{2}+m_{v}^{2}} \int_{-1}^{1} \mathrm{~d} u \mathscr{F}\left(E_{1}, E_{3}, u\right),
\end{aligned}
$$

where $k_{\max }=\sqrt{E_{1}^{2}+m_{v}^{2}}$ and we have used the identity

$$
\mathrm{d} k_{3} k_{3}=\mathrm{d} E_{3} E_{3}, \quad k_{3}=\sqrt{E_{3}^{2}+m_{v}^{2}} .
$$

The differential energy loss, for a particle traveling at velocity $v_{v} \approx c$, undergoing a decay with energy loss $E_{1}-E_{3}$, due to the energy-resolved decay rate $(\mathrm{d} \Gamma / \mathrm{d} E) \mathrm{d} E$, in time $\mathrm{d} t=\mathrm{d} x / c$, reads as follows:

$$
\mathrm{d}^{2} E_{1}=-\left(E_{1}-E_{3}\right) \frac{\mathrm{d} \Gamma}{\mathrm{d} E_{3}} \mathrm{~d} E_{3} \frac{\mathrm{d} x}{c} .
$$

Now we set $c=1$, divide both sides of the equation by $\mathrm{d} x$, and integrate over the energy $E_{3}$ of the outgoing particle. One obtains

$$
\frac{\mathrm{d} E_{1}}{\mathrm{~d} x}=-\int \mathrm{d} E_{3}\left(E_{1}-E_{3}\right) \frac{\mathrm{d} \Gamma}{\mathrm{d} E_{3}} .
$$

Hence, the energy loss rate is obtained as

$$
\begin{aligned}
\frac{\mathrm{d} E}{\mathrm{~d} x}= & -\frac{G_{F}^{2}}{4} \frac{1}{(2 \pi)^{4}} \int_{0}^{E_{1}} \mathrm{~d} E_{3} \sqrt{E_{3}^{2}+m_{\nu}^{2}}\left(E_{1}-E_{3}\right) \\
& \times \int_{-1}^{1} \mathrm{~d} u \mathscr{F}\left(E_{1}, E_{3}, u\right) .
\end{aligned}
$$

After a long and somewhat tedious integration, one finds the following expressions:

$$
\begin{aligned}
\Gamma & =\frac{1}{3} \frac{G_{F}^{2} m_{v}^{4}}{192 \pi^{3}} E_{1}, \\
\frac{\mathrm{d} E_{1}}{\mathrm{~d} x} & =\frac{1}{3} \frac{G_{F}^{2} m_{v}^{4}}{192 \pi^{3}} E_{1}^{2} .
\end{aligned}
$$

These formulas are valid for $E_{1} \gg m_{v}$, which is easily fulfilled for all neutrino masses $m_{\nu}$. There is no threshold energy; that is, formulas (27a) and (27b) are, in particular, valid in the range $E_{1} \gtrsim 1 \mathrm{eV} \gg m_{v}$. Parametrically, they are of the same order-of-magnitude as those given in [35] for (charged) lepton pair Cerenkov radiation, but the threshold is zero for the neutrino pair emission. Hence, neutrino pair emission is the dominant decay channel in the mediumenergy domain, for an oncoming tachyonic neutrino flavor eigenstate. 


\section{Discussion and Conclusions}

In principle, tachyonic spin- $1 / 2$ theories have a number of properties which make them more attractive than their spin-zero counterparts. One distinctive feature is that the mass parameters enter only linearly in the Lagrangian [26], thus preventing the vacuum from becoming manifestly unstable against tachyon-antitachyon pair production. Also, it has been possible to calculate the time-ordered product of field operators, which leads to the Feynman propagator of the tachyonic field $[26,37]$. One also observes that the generalized Dirac Hamiltonian for the tachyonic spin-1/2 fields is pseudo-Hermitian, so that it becomes possible to formulate the quantum dynamics of tachyonic wave packets without having to overcome unsurmountable challenges [14]. In [38], it has been argued that, in view of the small neutrino interaction cross sections, it would be difficult to transport information faster than the speed of light using a neutrino beam, if neutrinos are just a bit superluminal (tachyonic). The sign of the mass square of neutrinos has not yet been determined experimentally, in contrast to differences of mass squares among neutrino flavor eigenstates.

Here, we calculate the decay rate and energy loss rate, for a hypothetically tachyonic neutrino flavor, against neutrino pair Cerenkov radiation. It needs to be checked if the absence of a threshold would lead to a disagreement with high-energy data on neutrinos of cosmic origin. In fact, the IceCube experiment [31, 32] has observed 37 neutrinos having energies $E_{v}>10 \mathrm{TeV}$ during the first three years of data taking. Three of these events ("Ernie," "Bert," and "Big Bird") had energies $E_{v}>1 \mathrm{PeV}$, while "Big Bird" is famous for having an energy of $E_{v}=(2.004 \pm 0.236) \mathrm{PeV}$. A blazar has been identified as a possible source of this highly energetic neutrino [39]. Neutrinos registered by IceCube have to "survive" the possibility of energy loss by decay, and if they are tachyonic, then lepton and neutrino pair Cerenkov radiation processes become kinematically allowed.

The results given in (27a) and (27b) for the decay rate and energy loss rate due to neutrino pair Cerenkov radiation are not subject to a threshold energy; parametrically they are of the same order-of-magnitude as those given for lepton pair Cerenkov radiation in [35], but the threshold energy is zero. Let us estimate the relative energy loss due to neutrino pair Cerenkov radiation over a distance

$$
L=15 \times 10^{9} \mathrm{ly}=1.42 \times 10^{26} \mathrm{~m},
$$

assuming a (relative large) neutrino mass parameter of $m_{0}=$ $10^{-2} \mathrm{eV}$. One obtains the relative energy loss according to (27b),

$$
\frac{L}{E_{1}} \frac{\mathrm{d} E_{1}}{\mathrm{~d} x}=\frac{1}{3} \frac{G_{F}^{2} m_{0}^{4}}{192 \pi^{3}} E_{1} L=5.02 \times 10^{-20} E_{1} / \mathrm{MeV}
$$

This means that, even at the large "Big Bird" energy of $E_{\nu}=$ $(2.004 \pm 0.236) \mathrm{PeV}$, the relative energy loss over 15 billion light years does not exceed 5 parts in $10^{11}$, which is negligible.
The decay rate is obtained as follows (again, assuming that $\left.m_{v}=10^{-2} \mathrm{eV}\right)$ :

$$
\Gamma=\frac{1}{3} \frac{G_{F}^{2} m_{0}^{4}}{192 \pi^{3}} E_{1}=1.06 \times 10^{-37}\left(E_{1} / \mathrm{MeV}\right)(\mathrm{rad} / \mathrm{s}) .
$$

Even for "Big Bird," this means that the decay rate does not exceed $2.12 \times 10^{-28}(\mathrm{rad} / \mathrm{s})$, which is equivalent to a lifetime of $\sim 10^{20}$ years, far exceeding the age of the universe. The neutrino pair Cerenkov radiation process, even if threshold-less, has such a low probability due to the weakinteraction physics involved that it cannot constrain the tachyonic models. Indeed, even for relatively large tachyonic neutrino mass parameters of the order of $10^{-2} \mathrm{eV}$, and for the largest neutrino energies observed, the process leads only to a vanishingly small relative energy loss for an oncoming neutrino of cosmic origin 15 billion light years away. The lifetime of the tachyonic neutrino far exceeds the age of the universe. Our quick estimate shows that "Big Bird" would have survived the travel from the blazar PKS B1424-418 (see [39]). In other words, neutrino pair Cerenkov radiation does not pressure the tachyonic neutrino hypothesis.

We thus take the opportunity here to correct claims recently made by one of us (U.D.J.) in [35], where a hypothetical cutoff of cosmic neutrino spectrum at the Big Bird energy was related to the threshold energy for (charged) lepton pair Cerenkov radiation and thus to a neutrino mass parameter. In [35], it was overlooked that (i) a further decay process exists for tachyonic neutrinos which is not subject to a threshold condition and (ii) that the absolute value of both (charged) lepton and neutrino pair Cerenkov radiation is too small (both above and below threshold) to lead to any appreciable energy loss of an oncoming tachyonic neutrino flavor eigenstate, over cosmic distances and time scales. Hence, it is not possible, in contrast to the conclusions of [35], to relate the lepton pair threshold to the tachyonic mass parameter. The (more optimistic) conclusion thus is that neither lepton nor neutrino pair Cerenkov radiation processes pressure the tachyonic model.

However, for the Lorentz-violating models, important limits on the available parameter space have been set in $[33,34]$, based on (charged) lepton pair Cerenkov radiation alone. Roughly speaking, the reason for the pressure on the Lorentz-violating models is that even small Lorentz violations at $\mathrm{PeV}$ energies correspond to high "virtualities" of the superluminal particles and hence relatively large (energydependent) mass parameters. It is quite imperative that the additional decay process studied here should also be calculated for the different kinematic conditions in Lorentzviolating models, where it will further limit the available parameter space for the Lorentz-violating parameters. Note that, for example, employing a Lorentz-violating dispersion relation $E=v|\vec{p}|[29,30]$, with $v>1$, a quick calculation shows the absence of a neutrino pair production threshold in the Lorentz-violating model; the reason being simple: namely, one has $E \rightarrow 0$ for $|\vec{p}| \rightarrow 0$, and it thus becomes possible to generate Lorentz-violating neutrino pairs with near-zero four-momenta. The additional decay process uncovered here thus has the potential of fundamentally changing the bounds 
to be inferred for the Lorentz-violating parameters, from the cosmic high-energy neutrinos, within the Lorentz-violating models.

To conclude, the Lorentz-violating model is pressured at high energies, where even numerically tiny values of the Lorentz-violating parameters induce large deviations from the light-like dispersion relation, corresponding to a numerically large value of the "effective mass" $m_{*}$ with $E^{2}-$ $\vec{p}^{2}=m_{*}^{2}=\left(v^{2}-1\right) \vec{p}^{2}$ (where we assume the dispersion relation $E=v|\vec{p}|$ given in $[29,30])$. By contrast, the tachyonic model is fully compatible with astrophysical data collected at high energies, while the tachyonic dispersion relation predicts noticeable deviations from the speed of light only for comparatively low-energy neutrinos. A proposal to test the tachyonic hypothesis, in the low-energy domain, has recently been published in [38]. Finally, we also refer to [38] for clarifying remarks on general aspects of the tachyonic model.

\section{Conflicts of Interest}

The authors declare that there are no conflicts of interest regarding the publication of this paper.

\section{Acknowledgments}

The authors acknowledge helpful conversations with $\mathrm{R}$. Ehrlich. This research has been supported by the NSF (Grant PHY-1403937) and by a János Bolyai Research Scholarship of the Hungarian Academy of Sciences.

\section{References}

[1] O. M. Bilaniuk, V. K. Deshpande, and E. C. Sudarshan, “"Meta” relativity," American Journal of Physics, vol. 30, pp. 718-723, 1962.

[2] J. Dhar and E. C. G. Sudarshan, "Quantum field theory of interacting tachyons," Physical Review A: Atomic, Molecular and Optical Physics, vol. 174, no. 5, pp. 1808-1815, 1968.

[3] O.-M. Bilaniuk and E. C. G. Sudarshan, "Causality and spacelike signals," Nature, vol. 223, no. 5204, pp. 386-387, 1969.

[4] G. Feinberg, "Possibility of faster-than-light particles," Physical Review A: Atomic, Molecular and Optical Physics, vol. 159, no. 5, pp. 1089-1105, 1967.

[5] G. Feinberg, "Lorentz invariance of tachyon theories," Physical Review D: Particles, Fields, Gravitation and Cosmology, vol. 17, no. 6, pp. 1651-1660, 1978.

[6] E. Recami and R. Mignani, "Classical theory of tachyons (special relativity extended to superluminal frames and objects)," $L a$ Rivista del Nuovo Cimento Series 2, vol. 4, no. 3, p. 398, 1974.

[7] G. D. Maccarrone and E. Recami, "Two-body interactions through tachyon exchange," Il Nuovo Cimento A, vol. 57, no. 1 , pp. 85-101, 1980.

[8] D. G. Boulware, "Unitarity and interacting tachyons," Physical Review D: Particles, Fields, Gravitation and Cosmology, vol. 1, no. 8, pp. 2426-2427, 1970.

[9] T. Chang, "Parity Violation and Neutrino Mass," Nucl.Sci. Technol, vol. 13, pp. 129-133, 2002.

[10] E. Recami, "Superluminal waves and objects: an overview of the relevant experiments," Journal of Physics: Conference Series, vol. 196, Article ID 012020, 2009.
[11] O. M. Bilaniuk, “Tachyons," Journal of Physics: Conference Series, vol. 196, no. 1, Article ID 012021, 2009.

[12] S. K. Bose, "Aspects of Tachyon theory," Journal of Physics: Conference Series, vol. 196, Article ID 012022, 2009.

[13] A. Chodos, A. I. Hauser, and V. Alan Kostelecký, "The neutrino as a tachyon," Physics Letters B, vol. 150, no. 6, pp. 431-435, 1985.

[14] U. D. Jentschura and B. J. Wundt, "Pseudo-Hermitian quantum dynamics of tachyonic spin-1/2 particles," Journal of Physics A: Mathematical and Theoretical, vol. 45, no. 44, Article ID 444017, 2012.

[15] C. M. Bender and S. Boettcher, “"Real spectra in non-hermitian hamiltonians having PT-symmetry," Physical Review Letters, vol. 80, no. 24, pp. 5243-5246, 1998.

[16] C. M. Bender and G. V. Dunne, "Large-order perturbation theory for a non-Hermitian PT-symmetric Hamiltonian," Journal of Mathematical Physics, vol. 40, no. 10, pp. 4616-4621, 1999.

[17] C. M. Bender, S. Boettcher, and P. N. Meisinger, "PT -symmetric quantum mechanics," Journal of Mathematical Physics, vol. 40, no. 5, pp. 2201-2229, 1999.

[18] C. M. Bender and E. J. Weniger, "Numerical evidence that the perturbation expansion for a non-Hermitian PT -symmetric Hamiltonian is Stieltjes," Journal of Mathematical Physics, vol. 42, no. 5, pp. 2167-2183, 2001.

[19] C. M. Bender, D. C. Brody, and H. F. Jones, "Erratum: Complex Extension of Quantum Mechanics," Physical Review Letters, vol. 92, no. 11, pp. 119902-1, 2004.

[20] A. Mostafazadeh, "Pseudo-Hermiticity versus PT -symmetry: The necessary condition for the reality of the spectrum of a nonHermitian Hamiltonian," Journal of Mathematical Physics, vol. 43, no. 1, pp. 205-214, 2002.

[21] A. Mostafazadeh, "Pseudo-Hermiticity versus PT -Symmetry. II. A complete characterization of non-Hermitian Hamiltonians with a real spectrum," Journal of Mathematical Physics, vol. 43, no. 5, pp. 2814-2816, 2002.

[22] A. Mostafazadeh, "Pseudo-Hermiticity versus PT -Symmetry III: Equivalence of pseudo-hermiticity and the presence of antilinear symmetries," Journal of Mathematical Physics, vol. 43, no. 8, pp. 3944-3951, 2002.

[23] A. Mostafazadeh, "Pseudo-Hermiticity and Generalized PTand CPT-Symmetries," Journal of Mathematical Physics, vol. 44, no. 3, pp. 974-989, 2003.

[24] U. D. Jentschura, A. Surzhykov, and J. Zinn-Justin, "Unified treatment of even and odd anharmonic oscillators of arbitrary degree," Physical Review Letters, vol. 102, no. 1, Article ID 011601, 2009.

[25] U. D. Jentschura, A. Surzhykov, and J. Zinn-Justin, "MultiInstantons and Exact Results III: Unified De-scription of the Resonances of Even and Odd Anharmonic Oscillators," Annals of Physics, vol. 325, no. 5, pp. 1135-1172, 2010.

[26] U. D. Jentschura and B. J. Wundt, "From generalized dirac equations to a candidate for dark energy," ISRN High Energy Physics, vol. 2013, Article ID 374612, 21 pages, 2013.

[27] V. A. Kostelecký and R. Lehnert, "Stability, causality, and Lorentz and CPT violation," Physical Review D: Particles, Fields, Gravitation and Cosmology, vol. 63, no. 6, Article ID 065008, 2001.

[28] V. A. Kostelecký and M. Mewes, "Neutrinos with Lorentzviolating operators of arbitrary dimension," Physical Review D: Particles, Fields, Gravitation and Cosmology, vol. 85, Article ID 096005, 2012. 
[29] A. G. Cohen and S. L. Glashow, "Pair creation constrains superluminal neutrino propagation," Physical Review Letters, vol. 107, no. 18, Article ID 181803, 2011.

[30] F. Bezrukov and H. M. Lee, "Model dependence of the bremsstrahlung effects from the superluminal neutrino at OPERA," Physical Review D: Particles, Fields, Gravitation and Cosmology, vol. 85, no. 3, Article ID 031901, 2012.

[31] M. G. Aartsen, R. Abbasi, Y. Abdou et al., "First Ob-servation of PeV-Energy Neutrinos with IceCube," Physical Review Letters, vol. 111, Article ID 021103, 2013.

[32] M. G. Aartsen, M. Ackermann, J. Adams et al., "Observation of High-Energy Astrophysical Neutrinos in Three Years of IceCube Data," Physical Review Letters, vol. 113, Article ID 101101, 2014.

[33] F. W. Stecker and S. T. Scully, "Propagation of superluminal PeV IceCube neutrinos: a high energy spectral cutoff or new constraints on Lorentz invariance violation," Physical Review D: Particles, Fields, Gravitation and Cosmology, vol. 90, no. 4, Article ID 043012, 2014.

[34] F. W. Stecker, "Limiting superluminal electron and neutrino velocities using the 2010 Crab Nebula flare and the IceCube PeV neutrino events," Astroparticle Physics, vol. 56, pp. 16-18, 2014.

[35] U. D. Jentschura and R. Ehrlich, "Lepton Pair Čerenkov Radiation Emitted by Tachyonic Neutrinos: Lorentz-Covariant Approach and IceCube Data," Advances in High Energy Physics, vol. 2016, Article ID 4764981, 2016.

[36] C. Itzykson and J. B. Zuber, Quantum Field Theory, McGrawHill, New York, NY, USA, 1980.

[37] U. D. Jentschura and B. J. Wundt, "Localizability of tachyonic particles and neutrinoless double beta decay," The European Physical Journal C, vol. 72, no. 2, pp. 1-13, 2012.

[38] U. D. Jentschura, D. Horváth, S. Nagy, I. Nándori, Z. Trócsányi, and B. Ujvári, "Weighing the neutrino," International Journal of Modern Physics E, vol. 23, no. 1, Article ID 1450004, 2014.

[39] M. Kadler, F. Krauß, K. Mannheim et al., "Coincidence of a high-fluence blazar outburst with a PeV-energy neutrino event," Nature Physics, vol. 12, no. 8, pp. 807-814, 2016. 

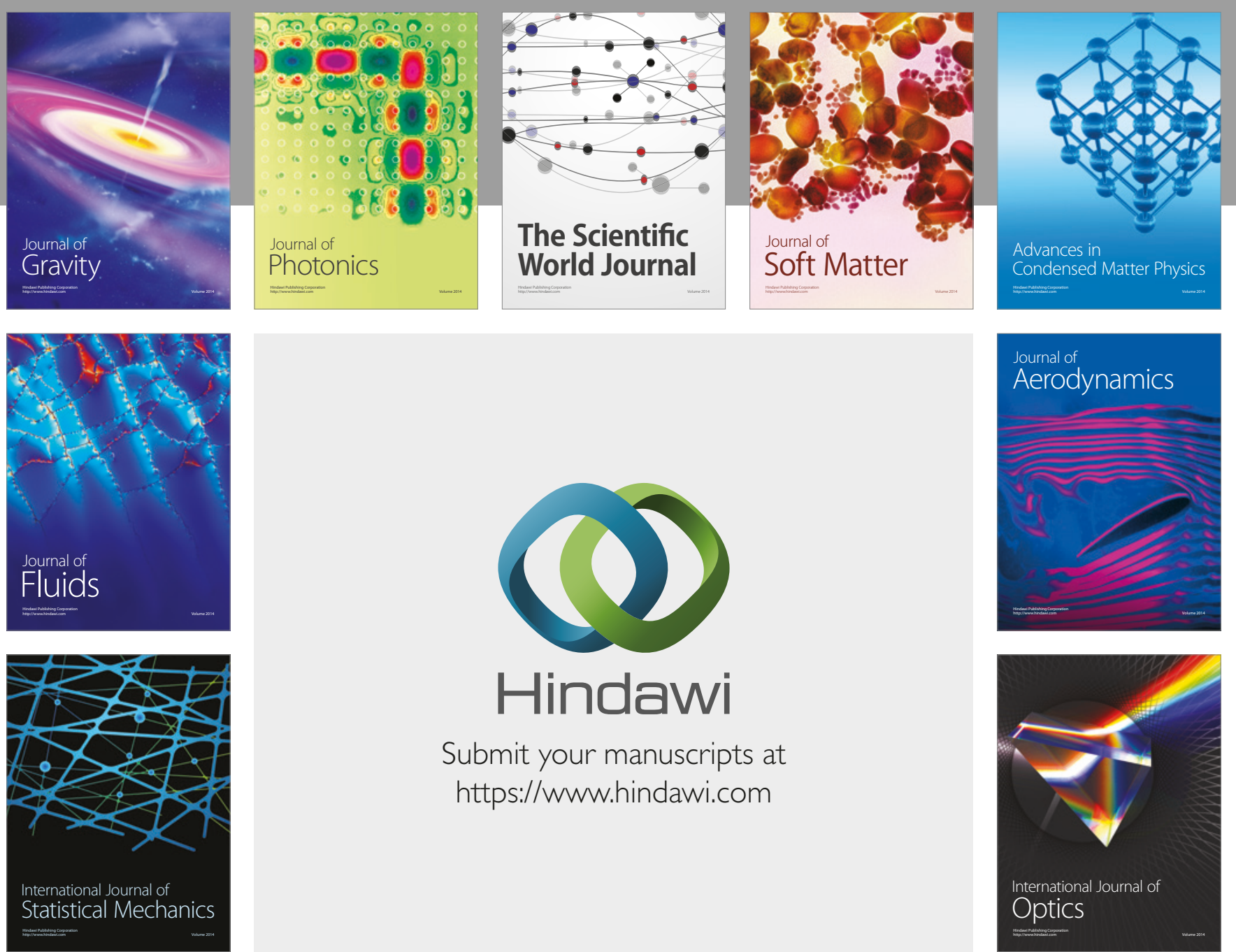

Submit your manuscripts at

https://www.hindawi.com
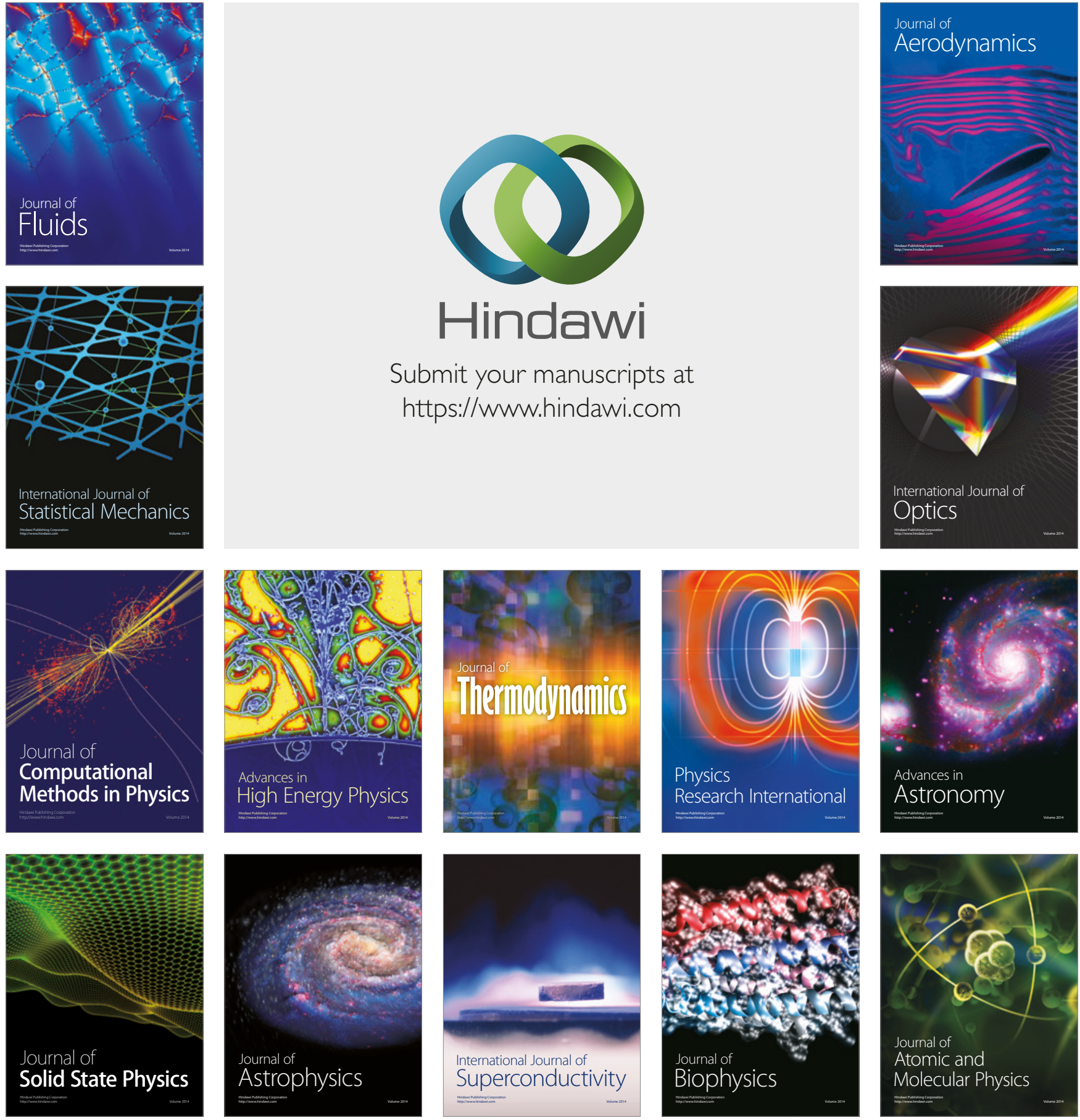